\title{
Efficacy of Infliximab Therapy for a Patient with Superficial Thrombophlebitis and Rheumatoid Arthritis
}

\author{
Masahiro Tada ${ }^{1}$, Kazuyoshi Fukai ${ }^{2}$, Tadashi Okano ${ }^{1}$, Yuko Sugioka ${ }^{1}$, Shigeyuki Wakitani ${ }^{1}$, \\ Hiroaki Nakamura', ${ }^{1}$ Tatsuya Koike ${ }^{3 *}$
}

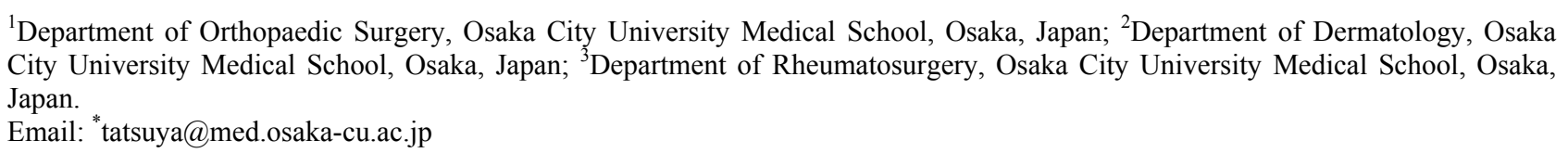

Received April 18 ${ }^{\text {th }}, 2011$; revised July 27 ${ }^{\text {th }}, 2011$; accepted August $23^{\text {th }}, 2011$.

\begin{abstract}
Superficial thrombophlebitis is known as a frequent complication of Behçet's disease. Infliximab may promote healing of superficial thrombophlebitis in patients with Behçet's disease. However, thrombophlebitis as a complication of rheumatoid arthritis $(R A)$ is rare and treatments have not been reported. We describe the case of a 47-year-old man with RA with complications of superficial thrombophlebitis who was treated using methotrexate and infliximab. Erythema nodosum and cord-like induration with pain in the extremities completely disappeared following a single infusion of infliximab and oral acetylsalicylic acid was not needed. This case suggests that infliximab might offer effective treatment for patients showing superficial thrombophlebitis with RA.
\end{abstract}

Keywords: Infliximab, Superficial Thrombophlebitis, Treatment, Rheumatoid Arthritis, Tumor Necrosis Factor $\alpha$

\section{Introduction}

The symptoms of superficial thrombophlebitis (STP) are skin redness, edema and cord-like induration with pain in the extremities. Pathologies known to be associated with STP include Behçet's disease (BD), infection, tuberculosis, tumor, sarcoidosis, ulcerous colitis, and antiphospholipid antibody syndrome [1]. BD needs to be considered in the differential diagnosis for patients showing the combination of STP and arthritis. However, when BD and other diseases associated with STP have been ruled out, rheumatoid arthritis (RA) should be considered. The combination of STP and RA is very rare, and treatments have not been reported.

Infliximab (IFX), a monoclonal antibody against tumor necrosis factor (TNF)- $\alpha$, has been approved for use in the treatment of ophthalmic lesions of BD [2-5] and for RA in combination with methotrexate (MTX) [6]. IFX effectively blocks the inflammatory process underlying BD and produces clinical improvements [7-10]. IFX treatment is known to be effective for STP with BD $[4,10]$, but whether IFX is effective against STP with RA remains unclear. We describe herein the case of a man with STP and RA who was successfully treated using IFX. IFX might be efficacious for STP in patients with RA.

\section{Case Report}

In May 2009, a 47-year-old man was referred to our clinic from a dermatologist due to joint tenderness and swelling in the wrists and fingers. He had symmetric polyarthritis of 4 joint areas, including the wrist, proximal interphalangeal (PIP), metacarpal phalangeal (MP) and ankle joints. He had a 2-month history of morning stiffness lasting around 2 hours, but no subcutaneous nodules were found. The patient had a past history of STP and had been prescribed acetylsalicylic acid (Bufferin; Eisai Pharmaceutical, Tokyo, Japan) at $162 \mathrm{mg}$ /day since 2005. Symptoms disappeared during two months of medication. At the same time as joint tenderness and swelling, he displayed erythema nodosum and cord-like induration with pain in the left forearm, lower leg, and chest (Figures 1(a) and (b)). Skin biopsy from the indurated cordlike nodule on the right chest revealed a re-penetrated vessel with thickened walls due to thrombosis and an 
occlusive vessel filled with leukocytes to remove thrombosis at the level of the deep dermis, suggesting a diagnosis of STP (Figure 2).

Examinations were performed to discriminate between 2 potential pathologies: RA or BD. Physical examination showed no genital or oral ulcerations or eye or gastrointestinal lesions suggestive of BD. Laboratory findings revealed that erythrocyte sedimentation rate (ESR) was elevated to $33 \mathrm{~mm} /$ hour with a C-reactive protein (CRP) level of $4.16 \mathrm{mg} / \mathrm{dl}$ and matrix metalloproteinase (MMP)-3 level of $126.1 \mathrm{ng} / \mathrm{ml}$. Serological examinations were negative for autoantibodies of rheumatoid factor, anti-cyclic citrullinated peptide and antinuclear antibodies. Results of human lymphocyte antigen (HLA)-B51 and anti-neutrophil cytoplasmic autoantibody were also negative. Radiography showed bone damage including erosions in bilateral wrists and left MP and PIP joints (Figure 3).

No evidence was seen of other diseases causing TP, such as infection, tuberculosis, tumor, sarcoidosis, ulcerous colitis, or antiphospholipid antibody syndrome. Because there had been negative pinprick skin test, no lymphadenopathy on chest x-ray, no gastrointestinal manifestations to suggest of inflammatory bowel disease and normal antiphospholipid serologies. RA was therefore diagnosed made according to the ACR (American College of Rheumatology) 1987 criteria for the classification of RA. In this case, RA and STP were present simultaneously. According to the Steinbroker classification, functional class was estimated as class I and stage was assessed as stage II.

The patient started treatment with salazosulfapyridine at $500 \mathrm{mg} /$ day, but immediately stopped treatment due to the appearance of itchy rash on the extremities and trunk. Next, he was prescribed bucillamine at $200 \mathrm{mg}$ /day, but developed fatigue and could not continue taking this medicine. To address the continued inflammation, he started methotrexate (MTX) at $6 \mathrm{mg} /$ week without adverse events. As joint tenderness and swelling in the wrists and fingers did not disappear, he received $300 \mathrm{mg}$ of IFX $(4.4 \mathrm{mg} / \mathrm{kg})$ at 0,2 and 6 weeks and every 8 weeks thereafter, in combination with MTX at $8 \mathrm{mg} / \mathrm{week}$.

Swelling and tenderness of bilateral MP, PIP and ankle joints due to RA decreased and disappeared after the first infusion of IFX. Before initiating treatment with IFX, laboratory evaluations revealed: white blood cell count,

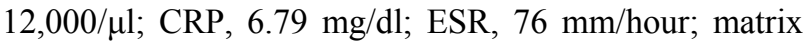
metalloproteinase (MMP)-3, $98.8 \mathrm{ng} / \mathrm{ml}$; disease activity score (DAS) 28-CRP4, 5.51; and DAS28-ESR4, 6.84. After 2 weeks of treatment (receiving only one infusion of IFX), CRP decreased to $0.06 \mathrm{mg} / \mathrm{dl}$, ESR decreased to $10 \mathrm{~mm} /$ hour, DAS28-CRP4 decreased to 1.97 and DAS28ESR4 decreased to 2.61. The patient was classified as a good responder according to European League Against
Rheumatism (EULAR) criteria and showed low disease activity. At the same time, STP disappeared completely from the left forearm, lower leg, and chest (Figure 1(c), (d)) and he did not have to take Bufferin.

As of the time of writing, 1 year after first treatment with IFX, erythema nodosum and cord-like induration
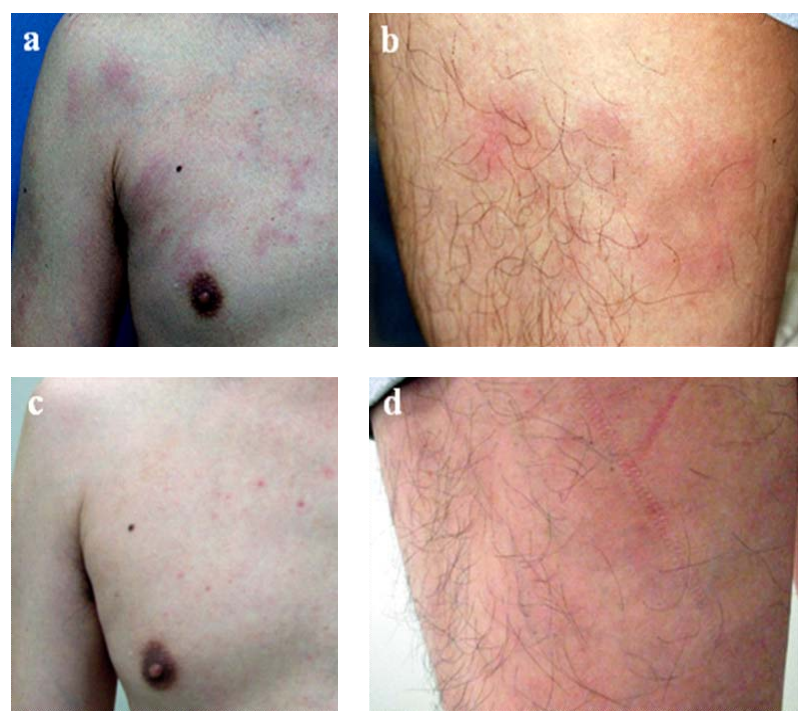

Figure 1. Superficial thrombophlebitis (STP) of front chest and lower leg. Before treatment of infliximab, he had erythma and cord-like induration with pain (a), (b). Lesion at two weeks after first treatment of infliximab. Immediately, STP disappeared completely in his front chest and lower leg (c), (d).
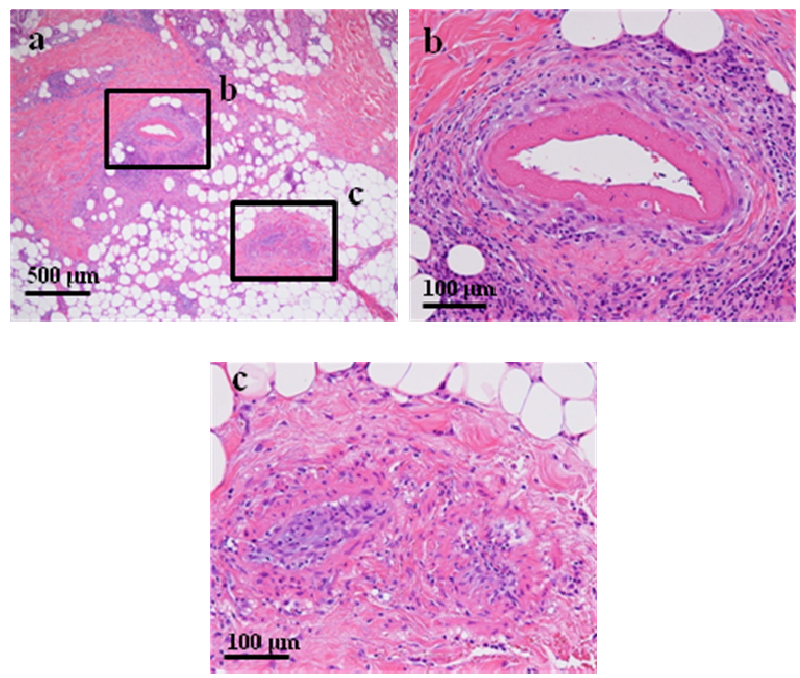

Figure 2. Skin biopsy from the indurated cord-like subcutaneous red nodule on the left chest revealed a re-penetrated vessel with the wall showing thick thrombosis (b), original magnification $\times 200$ ) and an occlusive vessel filled with leukocytes to remove the thrombosis (c), original magnification, $\times 200$ ). Lower magnification (a), original magnification, $\times 40$ ) shows that these vessels lie in the deep dermis. 


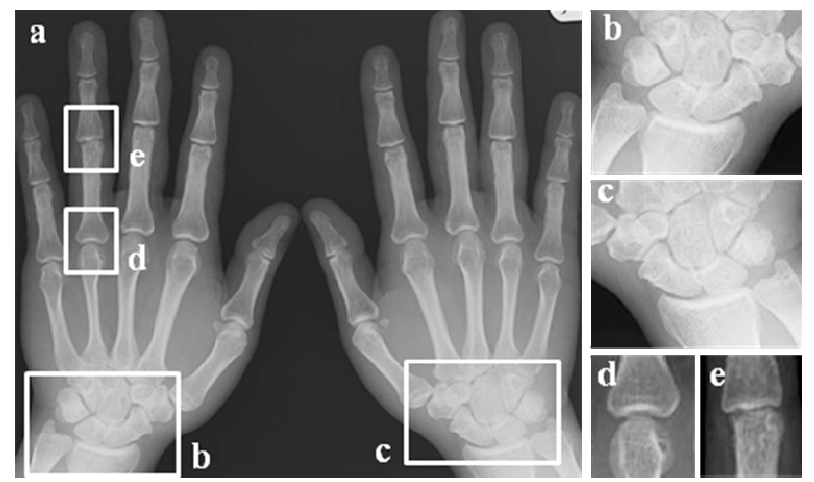

Figure 3. Radiography of bilateral hands (a). There are joint space narrowing in bilateral wrists (b), (c) and, joint space narrowing and erosions in left MP (d) and PIP joint (e) (4th finger).

have completely disappeared, RA remains very well controlled and disease activity is low. Maintenance treatment with IFX in combination with MTX has been continued to control RA and STP.

\section{Discussion}

This case report shows the benefit of IFX treatment for RA patients showing STP. Pathologies reportedly associated with STP include BD, infection, tuberculosis, tumor, sarcoidosis, ulcerous colitis, and antiphospholipid antibody syndrome. In this case, the patient had no symptoms of BD such as genital or oral ulcerations or eye or gastrointestinal lesions. STP was diagnosed based on the results of biopsy. Histopathological findings may be useful to diagnose STP. With reference to findings in STP, vessels were dilated and septa in fat tissues were sclerotic with mild infiltration of lymphohistiocytic cells. The patient showed joint swelling and tenderness in the wrists and fingers over 2 months, and fulfilled the ACR 1987 criteria. We thus diagnosed RA complicated with STP.

The combination of STP and RA is so rare that we were unable to identify any other reports of therapy. Conventional management of STP is medication and surgical treatment [11]. Medication includes non-steroidal anti-inflammatory drugs, anticoagulants and antibiotics. In this case, the patient had been prescribed acetylsalicylic acid in an effort to prevent STP. The reason why STP antedated RA diagnosis by almost 4 years is unknown. It might be an event caused by chance.

About the reason why STP may be prevalent in inflammatory conditions such as RA, we have considered the participation of cytokines in this situation and the cytokines should play an important role in STP progression.

Recently, a number of case reports have described good response of BD to treatment with IFX [7-10] or etanercept [12]. In particular, IFX therapy was reportedly effective and safe for long-term use according to an open-label, prospective, self-controlled study $[4,13,14]$. IFX therapy has been approved for use in the treatment of ophthalmic lesions of BD [2-5].

The effectiveness of IFX treatment for STP with BD has been reported $[4,10]$. Ohno et al. reported that erythema nodosum completely disappeared in 2 patients receiving $5 \mathrm{mg} / \mathrm{kg}$ of IFX [4]. Travis et al. reported thrombophlebitis resolved in 1 patient with use of IFX [10]. However, whether IFX therapy is effective against STP with RA has not been reported. In general, though, IFX therapy has been a great advance in the treatment of RA [6].

The standard dose of IFX has been $5 \mathrm{mg} / \mathrm{kg}$ for BD and MTX has not been used in combination. In this case, $300 \mathrm{mg}$ of IFX $(4.4 \mathrm{mg} / \mathrm{kg})$ was used in combination with MTX at $8 \mathrm{mg} /$ week. Continuation of IFX is likely to be necessary for suppression of disease progression of both RA and STP. The present case suggests that IFX might offer a potential effective therapy for RA patients with STP. Additional case studies and large prospective studies are needed to establish appropriate use of IFX for STP patients with complications of RA.

In conclusion, we report a case in which IFX therapy was used successfully for a patient with STP and RA. Administration of IFX may be useful for RA patients with STP.

\section{Acknowledgements}

Dr. Koike has received research grants and/or speaking fees from Takeda Pharmaceutical, Mitsubishi Tanabe Pharma Corporation, Chugai Pharmaceutical, Eisai, Abbott Japan, Teijin Pharma, Banyu Pharmaceutical and Ono Pharmaceutical. The other authors declare no conflict of interest.

\section{REFERENCES}

[1] L. Leon, A. D. Giannoukas, D. Dodd, P. Chan and N. Labropoulos, "Clinical Significance of Superficial Vein Thrombosis," European Journal of Vascular and Endovascular Surgery, Vol. 29, No. 1, 2005, pp. 10-17. doi:10.1016/j.ejvs.2004.09.021

[2] M. Accorinti, M. P. Pirraglia, M. P. Paroli, R. Priori, F. Conti and P. Pivetti-Pezzi, "Infliximab Treatment for Ocular and Extraocular Manifestations of Behcet's Disease," Japanese Journal of Ophthalmology, Vol. 51, No. 3, 2007, pp. 191-196. doi:10.1007/s10384-006-0425-y

[3] L. Niccoli, C. Nannini, M. Benucci, D. Chindamo, E. Cassara, C. Salvarani, L. Cimino, G. Gini, I. Lenzetti and F. Cantini, "Long-Term Efficacy of Infliximab in Refractory Posterior Uveitis of Behcet's Disease: A 24-Month Follow-up Study," Rheumatology (Oxford), Vol. 46, No. 7, 2007, pp. 1161-1164. 
doi:10.1093/rheumatology/kem101

[4] S. Ohno, S. Nakamura, S. Hori, M. Shimakawa, H. Kawashima, M. Mochizuki, S. Sugita, S. Ueno, K. Yoshizaki and G. Inaba, "Efficacy, Safety, and Pharmacokinetics of Multiple Administration of Infliximab in Behcet's Disease with refractory Uveoretinitis," The Journal of Rheumatology, Vol. 31, No. 7, 2004, pp. 1362-1368.

[5] K. F. Tabbara and A. I. Al-Hemidan, "Infliximab Effects Compared to Conventional Therapy in the Management of Retinal Vasculitis in Behcet Disease," American Journal of Ophthalmology, Vol. 146, No. 6, 2008, pp. 845850.

[6] P. E. Lipsky, D. M. van der Heijde, E. W. St Clair, D. E. Furst, F. C. Breedveld, J. R. Kalden, J. S. Smolen, M. Weisman, P. Emery, M. Feldmann, G. R. Harriman and R. N. Maini, "Infliximab and Methotrexate in the treatment of Rheumatoid Arthritis. Anti-Tumor Necrosis Factor Trial in Rheumatoid Arthritis with Concomitant Therapy Study Group," The New England Journal of Medicine, Vol. 343, No. 22, 2000, pp. 1594-1602. doi:10.1056/NEJM200011303432202

[7] G. Haugeberg, M. Velken and V. Johnsen, "Successful Treatment of Genital Ulcers with Infliximab in Behcet's Disease," Annals of the Rheumatic Diseases, Vol. 63, No. 6, 2004, pp. 744- 745. doi:10.1136/ard.2003.010975

[8] M. Naganuma, A. Sakuraba, T. Hisamatsu, H. Ochiai, H. Hasegawa, H. Ogata, Y. Iwao and T. Hibi, "Efficacy of Infliximab for Induction and Maintenance of Remission in Intestinal Behcet's Disease," Inflammatory Bowel Diseases, Vol. 14, No. 9, 2008, pp. 1259-1264. doi:10.1002/ibd.20457
[9] N. Pipitone, I. Olivieri, A. Padula, S. D’Angelo, A. Nigro, G. Zuccoli, L. Boiardi and C. Salvarani, "Infliximab for the Treatment of Neuro-Behcet's Disease: A Case Series and Review of the Literature," Arthritis \& Rheumatism, Vol. 59, No. 2, 2008, pp. 285-290. doi:10.1002/art.23345

[10] S. P. Travis, M. Czajkowski, D. P. McGovern, R. G. Watson and A. L. Bell, “Treatment of Intestinal Behcet's Syndrome with Chimeric Tumour Necrosis Factor Alpha Antibody," Gut, Vol. 49, No. 5, 2001, pp. 725-728. doi:10.1136/gut.49.5.725

[11] J. T. Lee and M. A. Kalani, "Treating Superficial Venous Thrombophlebitis," Journal of the National Comprehensive Cancer Network, Vol. 6, No. 8, 2008, pp. 760-765.

[12] M. Melikoglu, I. Fresko, C. Mat, Y. Ozyazgan, F. Gogus, S. Yurdakul, V. Hamuryudan and H. Yazici, "Short-Term Trial of Etanercept in Behcet's Disease: A Double Blind, Placebo Controlled Study," The Journal of Rheumatology, Vol. 32, No. 1, 2005, pp. 98-105.

[13] P. P. Sfikakis, P. H. Kaklamanis, A. Elezoglou, N. Katsilambros, P. G. Theodossiadis, S. Papaefthimiou and N. Markomichelakis, "Infliximab for Recurrent, Sight-Threatening Ocular Inflammation in Adamantiades-Behcet Disease," Annals of Internal Medicine, Vol. 140, No. 5, 2004, pp. 404-406.

[14] I. Tugal-Tutkun, A. Mudun, M. Urgancioglu, S. Kamali, E. Kasapoglu, M. Inanc and A. Gul, "Efficacy of Infliximab in the Treatment of Uveitis that Is Resistant to Treatment with the Combination of Azathioprine, Cyclosporine, and Corticosteroids in Behcet's Disease: An Open-Label Trial," Arthritis \& Rheumatism, Vol. 52, No. 8, 2005, pp. 2478- 2484. doi:10.1002/art.21231 